\title{
Search for Supersymmetry with photons
}

\author{
Krisztián Farkas ${ }^{a}$ \\ ${ }^{a}$ MTA-ELTE Lendület CMS Particle and Nuclear physics group, Eötvös Loránd University, \\ Pázmány Péter Sétány 1/A, Budapest, Hungary \\ E-mail: kfarkas@cern.ch
}

Most recent results on searches for supersymmetric (SUSY) particles decaying to final states containing high energy photons are presented. The searches are based on data collected at the centre-of-mass energy of $13 \mathrm{TeV}$ in proton-proton collisions recorded by the CMS detector.

The Eighth Annual Conference on Large Hadron Collider Physics -LHPC2020

25-30 May, 2020

online

The author wishes to thank for their support the Hungarian Academy of Sciences "Lendület" (Momentum) Program (LP 2015-7/2015) and the National Research, Development and Innovation Office of Hungary (K 124845). 


\section{Supersymmetric models}

Supersymmetric extensions of the standard model can solve many open questions in particle physics (e.g., dark matter, matter-antimatter assymetry). To decrease the number of free parameters of the Minimal Supersymmetric Standard Model (MSSM), specific SUSY breaking scenarios are considered. When the mediators of SUSY breaking from the hidden to the visible sector are gauge interactions, we arrive to Gauge Mediated Supersymmetry Breaking models (GMSB) with five new parameters or to its generalization, General Gauge Mediation (GGM) with eight new parameters.

In most models, R-parity conservation (RPC) is assumed to suppress baryon and lepton number violating processes. This implies that SUSY particles are produced in pairs, and the lightest supersymmetric particle (LSP) is stable, and if neutral, a good dark matter candidate.

In GMSB / GGM with RPC, the LSP is the gravitino (with a mass below $1 \mathrm{GeV}$ ) and the Next-to-LSP (NLSP) is typically a neutralino (sometimes mass-degenerate with a chargino) decaying via

$$
\tilde{\chi}_{0} \rightarrow \tilde{G}+\gamma / Z / H \quad\left(\tilde{\chi}^{ \pm} \rightarrow \tilde{G}+W^{ \pm}\right)
$$

Experimental results are interpreted in simplified models that consider only a single SUSY process with its cross-section $(\sigma)$, decay branching ratio (BR) and the masses of the SUSY particles considered as model parameters. Results on $\sigma \cdot \mathrm{BR}$ are then derived and can later be reinterpreted in specific models to provide constraints on their parameters.

\section{Experimental observables}

The CMS detector is described in Ref. [1].

The missing transverse momentum $\left(p_{T}^{\text {miss }}\right)$ measures the momentum imbalance of all observed physics objects. It can originate from neutrinos or (typically jet) momentum mismeasurements in SM processes, as well as from the weakly interacting LSP in SUSY models.

Hadronic activity (HT) is estimated by the scalar sum of jet transverse momenta. Large HT can come from the final state jets in strong SUSY production as illustrated on Fig. 1.

The kinematic quantities of the reconstructed photon $-p_{T}^{\text {miss }}$ system, such as the transverse energy sum and the transverse mass characterise the NLSP kinematics.

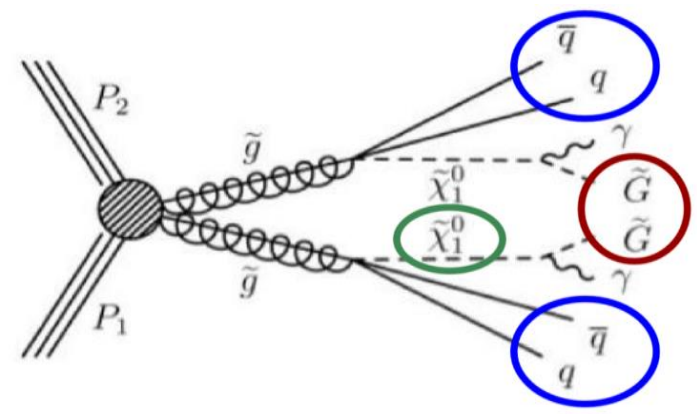

Figure 1. A typical Feynman diagram depicting gluino pair production followed by a cascade decay to gravitino. The gravitino appear as $p_{T}^{\text {miss }}$, then leaves the detector unseen, leading to a transverse momentum imbalance in the event $\left(\mathrm{p}_{\mathrm{T}}^{\mathrm{miss}}\right)$. The NLSP properties can be studied via the photon- $p_{T}^{\text {miss }}$ system. 


\section{Photon pair production}

The investigated process in this analysis is shown in Fig. 2. In this analysis [2], they were looking for a pair production of neutralinos, where Higgs boson decay to two photons via $\tilde{\chi}_{1}^{0} \rightarrow$ $H+\tilde{G}$ in the $H \rightarrow \gamma \gamma$ final state.

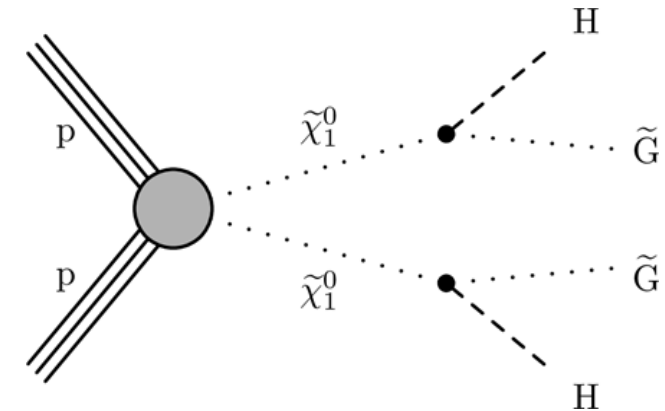

Figure 1. Feynman diagram of neutralino pair production in GMSB.

On the left side of Fig. 3 the signal extraction is illustrated. The green line represents a combined fit of the SUSY signal plus SM background hypothesis to the reconstructed diphoton mass distribution, while the blue line shows the total SM background (a side-band fit to diphoton mass distribution plus SM Higgs production from simulation), and the red line depicts the possible SUSY signal contribution (calculated as the combined fit result minus the SM background). The result is consistent with the SM without additional SUSY signal observed. Its interpretation in a GMSB motivated simplified model leads to an upper limit on the mass of a higgsino-like neutralino $m_{\widetilde{H}}>290 \mathrm{GeV}$ as shown on the right side of Fig. 3 .
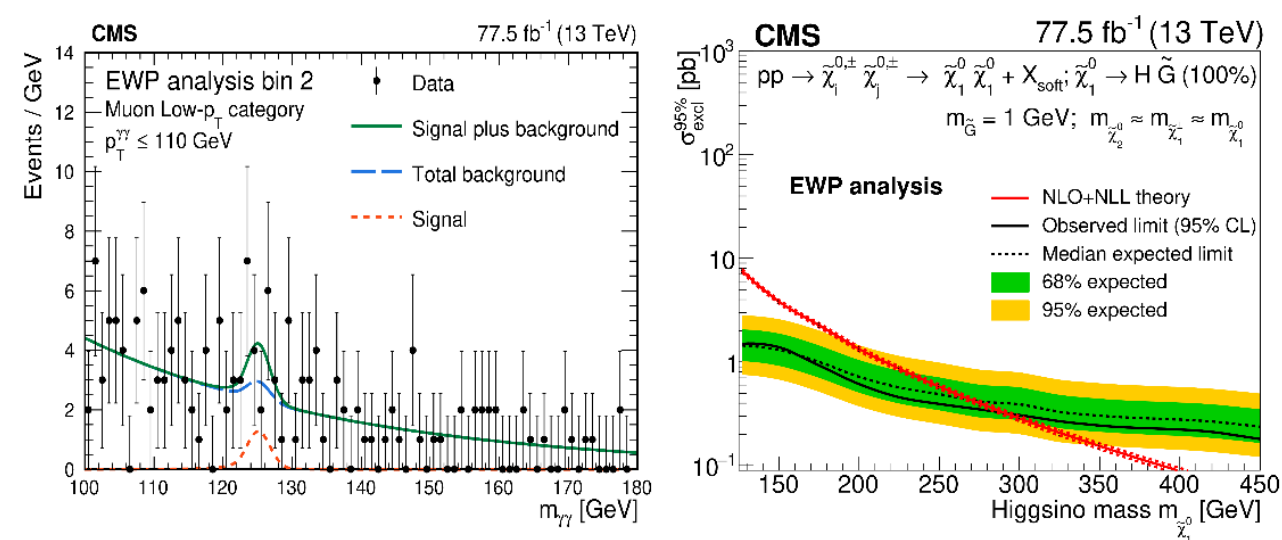

Figure 3. (left) The reconstructed diphoton mass distribution for an example search bin with the signalplus-background fit to illustrate the signal extraction procedure. (right) The observed and expected $95 \%$ CL upper limits on the production cross section for higgsino-like chargino-neutralino production together with the predicted theoretical cross-section as a function of the neutralino mass. [2]

\section{GGM combination}

CMS investigated the full GGM parameter space further by combining results from several analyses with photons $\left(\gamma \gamma+p_{T}^{\text {miss }}, \gamma+p_{T}^{\text {miss }}+\right.$ lepton, electroweak $\gamma+p_{T}^{\text {miss }}$, strong $\gamma+$ $\left.p_{T}^{\text {miss }}+\mathrm{HT}\right)$. A statistical combination of these analyses was done, after potential selection overlaps were removed. [3]. 
The expected 95\% CL exclusion areas in each included analysis and the gained area given by the combination can be shown on the GGM $\left(\mathrm{M}_{1}, \mathrm{M}_{2}\right)$ model parameter space (Fig. 4 left). And the results can be translated to physical SUSY sparticle masses and an exclusion limit can be given, as shown in the chargino-neutralino mass plane (Fig. 4 right). Limits are derived on the GGM parameters as well as on the physical SUSY particle masses. The combination improves sensitivity to sparticle masses by about $100 \mathrm{GeV}$. Observed mass limits are worse than expected due to an excess [4] in the diphoton category. The limit for the lightest chargino mass is $890 \mathrm{GeV}$ and for the gluino mass is $1950 \mathrm{GeV}$.
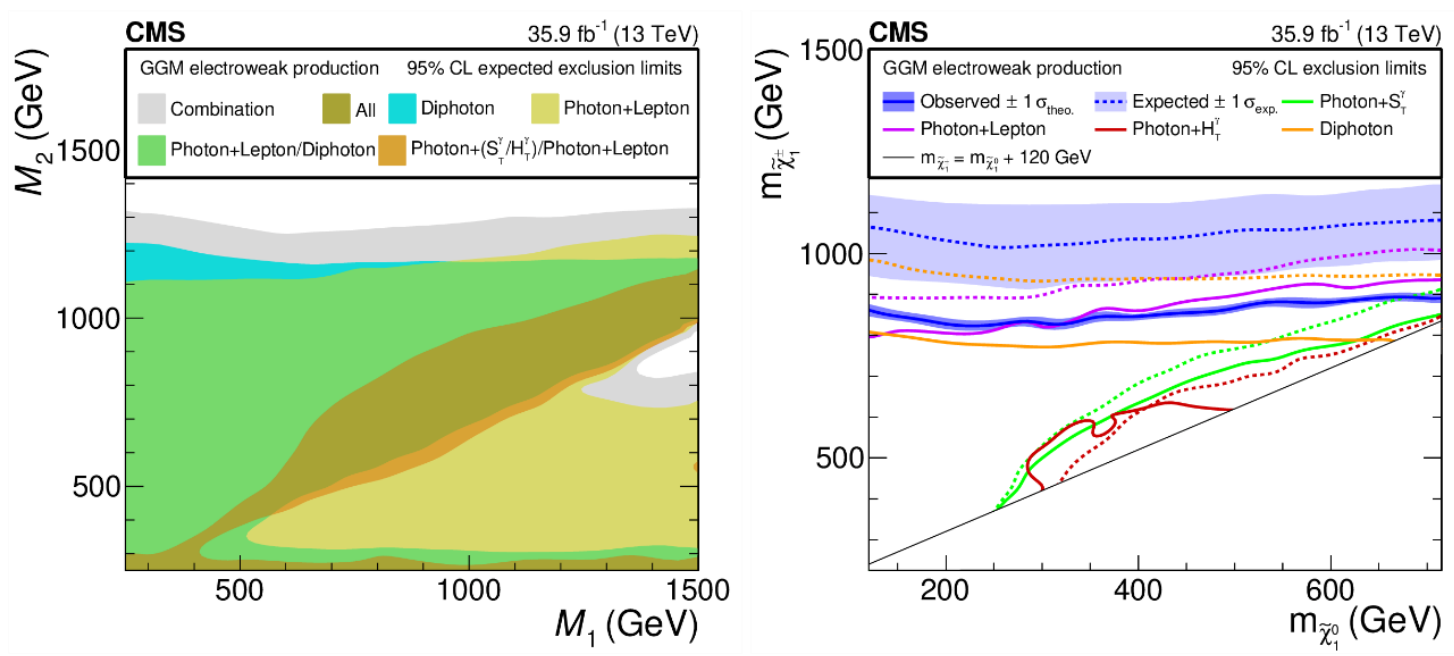

Figure 4. (left) The expected 95\% CL exclusion areas in the GGM scenario of the various analyses in terms of the GGM model parameters. (right) The observed and expected excluded areas in the physical neutralino and chargino mass plane. [3]

\section{Summary}

The presented searches address a large area of the GMSB (GGM) MSSM parameter space. Results so far are consistent with the SM (the largest excess of events is at the $2.4 \sigma$ level observed in the $\gamma \gamma+p_{T}^{\text {miss }}$ search). In the absence of a discovery, constraints are provided on SUSY model parameters and sparticle masses.

Work is ongoing to collect more data to reach the so far hidden corners of the SUSY parameter space, as only about $5 \%$ of the full planned LHC p-p integrated luminosity is recorded yet.

\section{References}

[1] CMS Collaboration, The CMS Experiment at the CERN LHC, JINST 3 (2008) S08004

[2] CMS Collaboration, Search for supersymmetry using Higgs boson to diphoton decays at $\sqrt{\mathrm{s}}=$ 13 TeV, JHEP 11 (2019) 109, arXiv: 1908.08500

[3] CMS Collaboration, Combined search for supersymmetry with photons in proton-proton collisions $\sqrt{s}=13 \mathrm{TeV}$, JHEP 11 (2019) 109, arXiv: 1907.00857

[4] CMS Collaboration, Search for supersymmetry in final states with photons and missing transverse momentum in proton-proton collisions at $13 \mathrm{TeV}$, JHEP 06 (2019) 143, arXiv:1903.07070 